\title{
High-density lipoprotein (HDL) metabolism and bone mass
}

\author{
Nicholaos I Papachristou1, Harry C Blair2,3, Kyriakos E Kypreos ${ }^{4}$ and \\ Dionysios J Papachristou'1,2
}

'Department of Anatomy-Histology-Embryology, Unit of Bone and Soft Tissue Studies, University of Patras Medical School, Patras, Greece

2Department of Pathology, University of Pittsburgh School of Medicine, Pittsburgh, Pennsylvania, USA 3Pittsburgh VA Medical Center, Pittsburgh, Pennsylvania, USA

${ }^{4}$ Department of Pharmacology, University of Patras Medical School, Patras, Greece
Correspondence should be addressed to D J Papachristou Email papachristoudj@med. upatras.gr

\begin{abstract}
It is well appreciated that high-density lipoprotein (HDL) and bone physiology and pathology are tightly linked. Studies, primarily in mouse models, have shown that dysfunctional and/or disturbed HDL can affect bone mass through many different ways. Specifically, reduced HDL levels have been associated with the development of an inflammatory microenvironment that affects the differentiation and function of osteoblasts. In addition, perturbation in metabolic pathways of HDL favors adipoblastic differentiation and restrains osteoblastic differentiation through, among others, the modification of specific bone-related chemokines and signaling cascades. Increased bone marrow adiposity also deteriorates bone osteoblastic function and thus bone synthesis, leading to reduced bone mass. In this review, we present the current knowledge and the future directions with regard to the HDL-bone mass connection. Unraveling the molecular phenomena that underline this connection will promote the deeper understanding of the pathophysiology of bone-related pathologies, such as osteoporosis or bone metastasis, and pave the way toward the development of novel and more effective therapies against these conditions.
\end{abstract}

\section{Key Words}

- adipose tissue

- apolipoprotein

- bone formation and resorption

- cholesterol

- skeletal biology

\section{Introduction}

Recent advances in the field of lipoproteins highlight a multifunctional role of high-density lipoprotein (HDL) in health and disease (Constantinou et al. 2015). HDL has been for decades an intriguing lipoprotein that attracted the attention of biomedical community, mainly because of its important role in atheroprotection (Constantinou et al. 2015). Indeed, the inverse correlation between HDL cholesterol (HDL-C) levels and the risk for developing coronary heart disease (Gofman et al. 1954, Havel et al. 1955, Miller et al. 1975, Tsompanidi et al. 2010, Kypreos et al. 2013, Karavia et al. 2014) suggested that high HDL-C levels in plasma are protective against the development of atherosclerosis. As a result, the majority of studies in the literature focused on the understanding of HDL-C levels in human pathology, a simplified approach to HDL that dates back to the early days when little was known about HDL structure and function. However, more recent data from experimental mice and clinical trials indicated that HDL particle functionality, as determined by its apolipoprotein (apo) and lipid content, is far more important in atheroprotection than HDL-C levels alone (Tsompanidi et al. 2010, Karavia et al. 2014, Constantinou et al. 2015). Even though HDL is usually referred to as the 'good cholesterol', certainly it is far more

Published by Bioscientifica Ltd. 
than just 'cholesterol'. HDL is rather a macromolecular assembly of proteins and lipids synthesized in the circulation as a result of a concerted action of apolipoproteins, lipid transporters and plasma enzymes. The main lipid cargo of mature HDL particles is esterified cholesterol. However, other lipids (phospholipids, sphingolipids, ceramides and so forth) are also part of HDL lipidome (Filou et al. 2016).

Though HDL was originally brought under the spotlight due its importance in protection from atherosclerosis, more recent findings propose a multifunctional role of HDL in numerous other biological processes including inflammation, oxidative stress, nitric oxide production and regulation of plasma glucose homeostasis (Karavia et al. 2014). In addition to atherosclerosis and coronary heart disease, recent data indicate that HDL may play a pivotal role in the pathology and treatment of other diseases including morbid obesity, non-alcoholic fatty liver disease, type 2 diabetes mellitus, obstructive pulmonary disease and numerous other diseases of the central nervous system (Constantinou et al. 2015). Recently, we identified a novel role of HDL in the pathogenesis of degenerative and metabolic bone diseases using experimental mouse models (Triantaphyllidou et al. 2013, Blair et al. 2016a), suggesting that low and dysfunctional HDL may contribute to an increased prevalence of these diseases by influencing molecular processes associated with bone synthesis and catabolism. Here, we review all new information pertinent to the effects of HDL and its major apolipoproteins on bone metabolism and function.

\section{Principles of HDL biogenesis and metabolism}

Recent data indicate that HDL is a mixture of lipoprotein particles with densities in the range of $1.063-1.21 \mathrm{~g} / \mathrm{mL}$, and depending on their lipid composition, these particles may assume a discoidal or spherical geometry. Mature spherical HDL particles contain approximately 45-55\% apoproteins, 26-32\% phospholipids, $15-20 \%$ esterified cholesterol, 3-5\% free cholesterol and approximately 5\% triglycerides (Tsompanidi et al. 2010). The main protein component of HDL is apolipoprotein A1 (APOA1) that plays a key role in the biogenesis and functions of HDL (Zannis et al. 2004). However, studies in mice showed that other apolipoproteins such as apolipoprotein E (APOE) (Kypreos \& Zannis 2007) and apolipoprotein CIII (APOCIII) (Kypreos 2008) are also capable of promoting the de novo biogenesis of HDL in a pathway similar to the one for the formation of APOA-I-containing HDL. Notably, APOA1-containing HDL appears to be structurally and functionally distinct from APOE-containing HDL particles (Filou et al. 2016).

Studies in cell cultures, as well as studies in experimental mouse models, showed that biogenesis of classical APOA1-containing HDL involves the lipid transporters ATP-binding cassette A1 (ABCA1) and G1 (ABCG1) and the plasma enzyme lecithin:cholesterol acyl transferase (LCAT) (Soutar et al. 1975, Chroni et al. 2003, Fitzgerald et al. 2004). In the early steps of HDL formation, lipid-free APOA1 that is secreted at about $70 \%$ by the liver (Timmins et al. 2005) and 30\% by the intestine (Brunham et al. 2006) interacts functionally with a dimeric form of the lipid transporter ABCA1 to acquire phospholipid and cholesterol (Nagata et al. 2013), thus forming a minimally lipidated APOA1. Through a series of intermediate steps that involve ABCG1, minimally lipidated APOA1 gradually forms discoidal HDL particles, which are then converted into spherical particles by plasma enzyme lecithin:cholesterol acyl transferase (LCAT) (Soutar et al. 1975). APOA1 on both discoidal and spherical HDL particles is then capable of interacting with scavenger receptor class B type I (SRBI) present on the surface of cells (Krieger 2001, Liu et al. 2002, Van Eck et al. 2005) to deliver cholesterol esters to the cell. Studies in mice support that the interactions of APOA1 on HDL with cell-surface SRBI are important for the atheroprotective functions of HDL (Trigatti et al. 2003). Additional steps in the metabolism of HDL involve its further processing by plasma enzyme cholesteryl ester transfer protein an enzyme that mediates the exchange of HDL cholesteryl esters with VLDL (very low-density lipoprotein) triglycerides. This step contributes to the reduction of HDL particle size, making it a more suitable substrate for SRBI, and the eventual catabolism of HDL cholesteryl esters by the LDL receptor through receptormediated uptake of cholesteryl ester-rich LDL. Additional processing of HDL in circulation involves the hydrolysis of its phospholipids and triglycerides by various lipases (lipoprotein lipase, hepatic lipase and endothelial lipase) and the transfer of phospholipids from VLDL/LDL to HDL by phospholipid transfer protein (Zannis et al. 2004).

Proteomic analyses revealed a diversity in HDL proteome that depending on the method of HDL isolation, includes more than 85 different proteins (Karlsson et al. 2005a,b, Heller et al. 2005, Rezaee et al. 2006, Vaisar et al. 2007, Shah et al. 2013), in addition to APOA1, APOE and APOCIII. Moreover, these studies showed that the plasma abundance of HDL-associated proteins is insufficient

Published by Bioscientifica Ltd. 
to permit one copy of protein for each HDL particle, suggesting that different proteins may be associated with different HDL particles that are differentially distributed across the HDL density spectrum (Tsompanidi et al. 2010). More recent data indicate that HDL proteome dictates its lipidome and subsequently HDL particle functionality (Filou et al. 2016), suggesting that the understanding of HDL proteome and the factors affecting it, are crucial steps in successfully improving HDL functionality.

\section{Biogenesis and function of bone cells}

\section{Osteoblast, the bone-forming cell and the 'osteoblast family'}

Bone is a form of specialized connective tissue that performs several essential functions including mechanical support for soft tissues, protection of internal organs, calcium homeostasis and support of haematopoiesis. The maintenance of bone mass in mammals depends upon the fine balance between bone synthesis and resorption, two vital tasks accomplished by osteoblasts and osteoclasts, respectively. Continuous bidirectional cross-talk between osteoblasts-osteoclasts is coordinated in time and space through a process that is fundamental for bone homeostasis and is called 'bone remodeling' (Fig. 1). This process is under the strict control of autocrine, paracrine and endocrine signals and is essential for the adaptation of bone to the mechanical loading and for the repair after damage (Sims \& Gooi 2008). Most importantly, disturbance in the coupling between bone synthesis and/ or bone degradation is the leading cause for numerous bone-related pathologies, including osteoporosis.

In the present review, we refer to bone cells in the context of the air-breathing vertebrates. In this context, bone is a complex tissue with advanced features not found in the skeleton of vertebrate phylogenetic precursors such as fish. Bone usually replaces solid and avascular mesenchymal tissues namely mineralized cartilage or fibrocartilage. Indeed, during bone formation in vitro, cartilage genes are invariably induced at early stages, suggesting that cartilage differentiation is required for osteoblasts formation (Blair et al. 2016b). This process is more profound in conditions where endochondral ossification is involved, such as normal long bone growth or fracture healing.

Osteoblasts are metabolically active cells, and as such, they possess large nucleus containing 2-4 nucleoli, very rich rough endoplasmic reticulum and Golgi apparatus. The name 'osteoblast' has its origins from the Greek

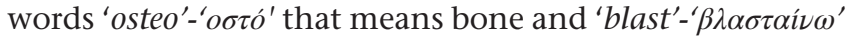
that means 'been born'. These cells are responsible for the production of the extracellular matrix that is composed mainly of collagen type 1 , with smaller amounts of specialized proteins including osteocalcin (Blair et al. 2007). Osteoblasts are under the tight control of paracrine, autocrine and endocrine signals. In fact, virtually all common signal transduction pathways converge on osteoblasts regulating their homeostasis (de Gorter \& ten Dijke 2013).

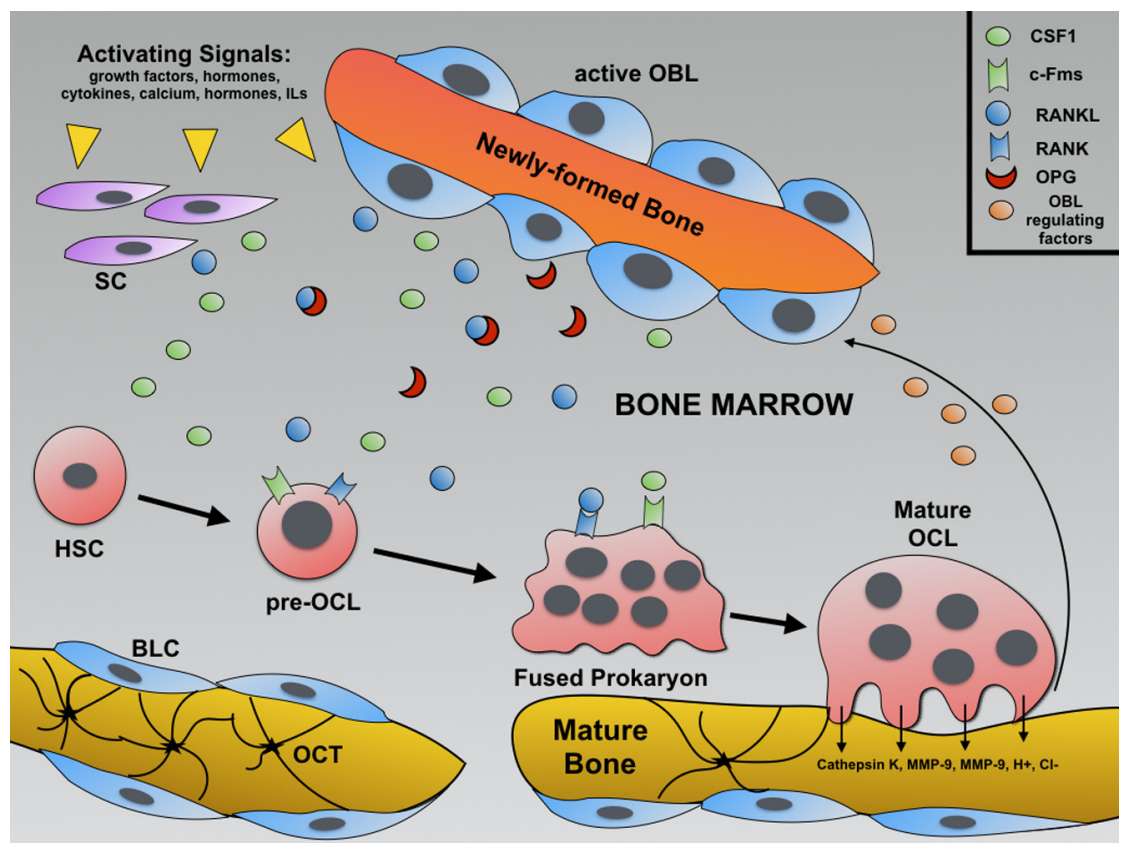

Figure 1

Diagram depicting the growth factors, cytokines and receptors that are involved in the 'coupling' between osteoblasts and osteoclasts and the regulation of osteoclast maturation and bone resorption. These phenomena that take place in the bone marrow are described in detail in the text. It should be noted that mature osteoclasts should adhere tightly to bone surfaces (mainly through $\alpha \nu \beta 3$ intern adhesions) to accomplish bone resorption. Moreover, note that the activated osteoblasts are large, cuboidal cells, with hefty nucleus; on the contrary, inactive bone lining cells are spindle shaped, with small elongated nucleus. Under specific stimuli, bone lining cells become metabolically active osteoblasts, regaining their bone-producing capacity. BLC, bone lining cells; CSF1, colony stimulation factor-1; HSC, hematopoietic stem cells; IL, interleukin; MMP, matrix metalloproteases; $\mathrm{OBL}$, osteoblasts; $\mathrm{OCL}$, osteoclasts; OCT, osteocyte; OPG, osteoprotegerin; RANK, receptor activator for nuclear factor $\kappa B$; RANKL, RANK-ligand; SC, stromal cells. 
New osteoblasts differentiate from mesenchymal stem cells in a skillfully coordinated fashion at the bone surfaces. Key transcription factors for osteoblast differentiation are osterix and RUNX2; the former is often used to regulate cre recombinase for osteoblast-specific gene expression (Blair et al. 2008). The molecular pathways that are responsible for osteoblast differentiation, proliferation and ultimately apoptosis are complex (de Gorter \& ten Dijke 2013). Among the most thoroughly studied and potent regulators of osteoblast differentiation are the mitogen-activated kinases (MAPK), the Wnt/ $\beta$-catenin, the bone morphogenic proteins (BMP) and transforming growth factor- $\beta$ (TGF $\beta$ ), the hedgehog, the Notch, the insulin-like growth factor 1 (IGF1), the fibroblast growth factor (FGF) and the calcium signal transduction pathways that ultimately converge on RUNX2, which is the master regulator of osteoblast differentiation (Blair et al. 2016b). The osteoblasts are connected through tight junctions, and gap junctions consisted mainly of connexin- 43 . The tight junctions exclude low molecular matrix materials, whereas bone is alive. Notably, however, this is not the case for dead bone, which is immediately permeable (Eberhardt et al. 2001). The most common bone formation defects, usually include mutations in type I collagen (osteogenesis imperfecta) (Forlino \& Marini 2016). There are many causes of low or high bone mass that are secondary to defects in specific factors, including serum lipids and lipoprotein receptors, as discussed elsewhere in this review.

Except from the bone-forming cells, the 'osteoblast family' also includes the bone lining cells and the osteocytes. Bone lining cells are bone surface-residing cells that are spindle shaped and metabolically and functionally quiescent. These cells separate bone matrix from the tissue extracellular fluid, creating a type of a tight 'epithelium'. The separation of matrix and extracellular space is an essential feature of bone, which is often not appreciated. Under the influence of specific signals, bone lining cells can regain their bone-forming capacity and produce bone. As osteoblastic function progresses and bone matrix expands, surface osteoblasts are eventually incorporated into the matrix that they have produced being transformed to osteocytes, the third member of the 'osteoblast family' (Bonewald 2011). Osteocytes are permanent components of living bone and the most abundant cells in human skeleton. Indeed, in the fully mature skeleton, osteocytes compose the $90-95 \%$ of the total number of bone cells, which is enormous given that osteoblasts constitute only the 4-6\% (Bonewald 2011). As these cells are entrapped in mineralized bone matrix, they harbor a delicate network of cell membrane processes to communicate with each other and with the bone surface cells. These processes contain connexin- 43 and run in small channels, the canaliculi, which permeate the extracellular matrix (Blair et al. 2016b). For years, it was believed that osteocytes are terminally differentiated cells with a small impact on skeletal metabolism. Nevertheless, it is now clear that the osteocyte network is surprisingly active, serving as an endocrine unit with key roles in bone remodeling and homeostasis (Bonewald 2011).

One of the most important functions of skeleton is to receive and respond to mechanical stimuli, primarily related to gravity and movement. Osteocytes are the most mechanosensitive cells of bone. Indeed, their large number and the perfectly developed cell-cell and cell-surface communications render them capable of receiving mechanical cues, which, in turn, are converted to biochemical and ultimately molecular response, through a finely tuned but extremely complex process called mechanotransduction (Papachristou et al. 2009, Thompson et al. 2012). Another worth-mentioning function of osteocytes is the production of sclerostin (Delgado-Calle et al. 2016), a protein that inhibits further growth of bone. Other important signals produced by osteocytes and osteoblasts are mediated by fibroblast growth factor 23 (FGF23), a specialized fibroblast growth factor family protein, which responds to 1,25-dihydroxyvitamin D3 and reduces phosphate reabsorption in the kidney (Huang et al. 2013).

\section{Osteoclasts, the bone-degrading cells}

Osteoclasts are multinucleated cells derived from monocyte family precursors (Blair et al. 2008), in sharp contrast to osteoblast that are of mesenchymal origin. Osteoclast precursors are mononucleated cells that during the process of differentiation and under the influence of specific factors that will be mentioned briefly later in this review, fuse forming the mature multinucleated cells, capable of bone resorption (Fig. 1). The name

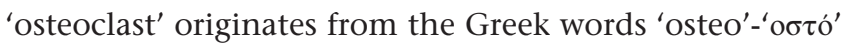
that means bone and 'clast' that means cut/destroy. As their name defines, the main function of these cells is the degradation of bone extracellular matrix. To accomplish this function, osteoclasts adhere tightly to bone surfaces through specialized integrin (mainly $\alpha \nu \beta 3$ ) adhesions. Acid-secreting H+-ATPase, which resides at their apical surfaces, facilitates bone mineral resorption by adding acid to it, solubilizing phosphate and calcium (Blair et al. 1989). The acid secretion is supported by chloride-proton

Published by Bioscientifica Ltd. 
exchange and chloride channels (Schlesinger et al. 1997, Palagano et al. 2015). Osteoclasts secrete acid proteinases, including cathepsins $\mathrm{B}$ and $\mathrm{K}$ and matrix metalloproteases (MMP) -9 and -13 that degrade collagen. Removal of bone components is achieved mainly by vacuolar translocation (Blair et al. 2008). As with other macrophage family cells, osteoclasts are dependent upon the macrophage colonystimulation factor-1 (CSF1), via the tyrosine kinase receptor c-fms, for survival and differentiation (Pixley \& Stanley 2004, Blair et al. 2005). CSF1 is produced by mesenchymal cells including osteoblasts and stromal cells and is responsible for the differentiation proliferation and survival of the committed cells and their progression to committed pre-osteoclasts (Fig. 1). Osteoclast precursors also express the receptor RANK (receptor activator for nuclear factor $\mathrm{\kappa B}$ ) that regulates the maturation of mono- and multi-nucleated pre-osteoclasts through its binding with RANK ligand (RANKL). RANKL is a type II homotrimeric transmembrane protein produced by osteoblast and stromal cells and is also secreted by activated T cells (Martin \& Sims 2015). In addition to RANKL, osteoblasts and stromal cells also produce and secrete osteoprotegerin (OPG), a soluble RANK decoy receptor, which prevents RANK from acting at distant sites as a scavenger and pharmacologically is a useful inhibitor of osteoclast formation (Boyle et al. 2003, Martin \& Sims 2015). Increased RANKL/OPG ratio promotes osteoclast formation and thus bone resorption (Fig. 1).

Primary osteoclast defects are rare, usually including defects in acid secretion, acid proteinases and cellular adhesion (Blair et al. 2004).

There is a tremendous amount of additional information on osteoclast differentiation, too much for a general review. Molecules including pituitary hormones, sex steroids, glucocorticoids, specific cytokines and growth factors are implicated in the regulation of bone formation and degradation (Blair et al. 2016b); nevertheless, our appreciation of the overall physiological control of bone homeostasis is still vague.

\section{Obesity, bone marrow fat, HDL and bone metabolism}

The past few years there is gradually increasing volume of data documenting a strong connection between fat and bone metabolism (Papachristou \& Blair 2016). Indeed, it has been demonstrated that disturbances in lipid metabolic pathways differentially affect bone cells resulting in the development of skeletal pathologies.
In this vein, a large number of epidemiological and animal studies have attempted to explore the association between obesity and bone mass. Notably, however, the results are contrasting, and it is not clear whether obesity has a positive or negative effect on bone mass. As a matter of fact, several, mainly older studies, suggested that obesity in terms of increased body weight has a protective role against bone loss and osteoporosis development (Reid et al. 1994, Reid 2008). This notion was mainly supported by the fact that increased body weight results in augmented mechanical stimulation on bone, which promotes osteoblast activation and bone formation, whereas on the other hand, it inhibits osteoclastogenesis and bone resorption (Papachristou et al. 2009). From a metabolic standpoint, obesity is associated with the secretion of the pancreatic hormones insulin, resistin and amylin, as well as with increased estrogen levels, molecules that serve as critical regulators of bone metabolism (Barsh et al. 2002). In addition, visceral adipocytes can secrete adipokines, and in particular leptin, adiponektin and resistin that also variably affect bone cells (Barsh et al. 2002). However, more recent studies have shown that the incidence of osteoporosis and bone fractures is increased in obese individuals compared to that in individuals within normal weight (Cao 2011). In this line, a more recent study on healthy premenopausal women showed that central adiposity is strongly associated reduced bone quality, stiffness and bone formation rate. In addition, epidemiological studies in humans propose that fat mass determines the quality of bone in a manner that is independent of body weight, in postmenopausal women (Reid et al. 1994). One possible explanation for this is that the local phenomena that take place within bone marrow have strong effect on bone cell functions, and thus, on bone mass, that possibly overcomes the impact of bone-acting circulating hormones. Indeed, conditions that are characterized by increased visceral adiposity are associated with increased bone marrow fat and reduced bone mass (Ng \& Duque 2010) (Fig. 2). The increased bone marrow adiposity that is observed in these conditions is primarily attributed to the fact that osteoblasts and bone marrow adipocytes that have common progenitor, the mesenchymal stem cell, represent the two sides of the same coin. Therefore, increased bone marrow adiposity is accompanied by decreased number of osteoblasts and vice versa (Reid 2008).

The reduced bone mass observed in obese individuals is also linked to the fact that obesity elicits low-grade inflammatory response, primarily mediated by the proinflammatory cytokines tumor necrosis factor- $\alpha$ (TNF $\alpha$ ),

Published by Bioscientifica Ltd. 


\title{
HDL and Bone Mass
}

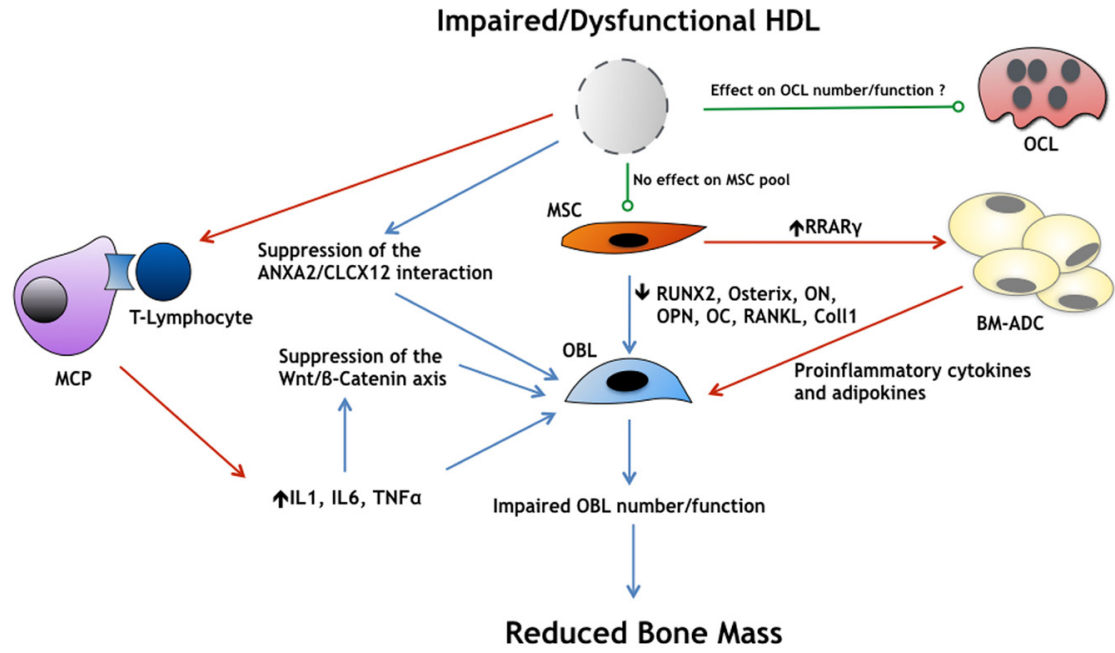

\begin{abstract}
Figure 2
This diagram shows the molecular mechanisms that underline the effect of impaired and/or dysfunctional HDL on bone mass. The red arrows represent the 'positive' effects, the blue arrows represent the 'negative' effects and the green arrows are indicative of 'no' or 'undefined' effect. ANXA2, Annexin-2; BM-ADC, bone marrow adipocytes; CLCX12, CXC chemokine ligand 12; Coll, collagen; IL, interleukin; MCP, macrophages; MSC, mesenchymal stem cells; OC, osteocalcin; $\mathrm{OCL}$, osteoclast; OBL, osteoblast; ON, osteonectin; OPN, osteopontin; RANK, receptor activator for nuclear factor $\mathrm{\kappa B}$; RANKL, RANK Ligand; TNF $\alpha$, tumor necrosis factor alpha.
\end{abstract}

interleukine-6 (IL6) and IL1 $\beta$ (Okin et al. 2013) and affects bone formation-resorption equilibrium and hence bone mass. Activated $\mathrm{T}$ lymphocytes can directly bind macrophages, triggering the production and secretion of the aforementioned cytokines. Under normal conditions, HDL inhibits the T lymphocyte-macrophage interaction preventing the initiation of the inflammatory response (Burger et al. 2002). It is now well appreciated that inflammation has a strong impact on bone remodeling differentially affecting the function of both the osteoblasts and the osteoclasts and thus possesses a central role in the development of bone-related metabolic pathologies, such as osteoporosis (Redlich \& Smolen 2012). Osteoblastic differentiation and activation are under the strict control of the Wnt/ $\beta$-catenin signaling axis. Briefly, this axis is activated when extracellular Wnt binds to a specific co-receptor complex composed of a member the Frizzled family of receptors (FZD) and the extracellular domain of the LDL receptor-related proteins (LRPs) -5 and -6 (in vertebrates) (Pandur \& Kuhl 2001). Among the common substrates of the Wnt/ $\beta$-catenin cascade, which is referred as the 'canonical Wnt pathway', are RUNX2, COX2, parathyroid hormone (PTH) and Indian hedgehog (IHH), crucial regulators of osteoblastic physiology. In an inflammatory background, TNF $\alpha$, IL6 and IL1 $\beta$ activate Dickkopf, sclerostin and secreted frizzled-related proteins (sFRP), which impede the formation of the WntLRPs-FZD assembly and hence suppress the anabolic downstream effects of the Wnt/ $\beta$-catenin pathway on osteoblasts (Clevers 2006). Another mechanism that is responsible for the inflammation-related bone loss is the activation of the IL6-JAK-STAT ((JUN N-terminal kinase)/ (signal transducers and activators of transcription)) signal transduction pathway that restrains the activation of the mitogen-activated protein kinase (MAPK)-AP1 signaling cascade, which in turn regulates the expression of the osteoblastogenic factors RUNX2, Osterix, Collagen type 1, Osteocalcin, PTHrP and OPG (Krum et al. 2010, Redlich \& Smolen 2012). A similar effect is evoked via the activation of the TNF-SMAD ubiquitylation regulatory factor (SMURF) 1/2 cascade that also deteriorates the expression of osteoblast regulators (Kaneki et al. 2006).

The effect of low-grade inflammation is also pronounced on osteoclasts. More specifically, activated $\mathrm{T}$ lymphocytes express RANKL and therefore have the ability to directly bind and activate osteoclast precursor cells, via the RANK-RANKL axis (Devlin et al. 1998, Ma et al. 2004). In addition, pro-inflammatory molecules secreted by the bone marrow adipocytes can enhance osteoclasts differentiation and activation in a RANK/ RANKL-independent fashion (Kobayashi et al. 2000). Inflammation is also associated with the production of the monocyte chemoattractant protein 1 (MCP1), a pro-inflammatory cytokine that is also implicated in the transcription of osteoclast-specific genes, primarily through the JAK-STAT signaling cascade (Redlich \& Smolen 2012). Nevertheless, less is known about the direct effect of HDL on osteoclasts physiology, a research field that is under scrutiny (Fig. 2).

In an effort to shed light into the effect of dietinduced obesity on bone mass, a recent study on adult mice that were fed high-fat diet for 11 weeks uncovered 
that bone responds to increased body weight in a bimodal fashion. Initially, obesity has an anabolic effect on bone due to enhanced mechanical loading and/or secretion of adipokines or growth factors that augment bone synthesis. Remarkably, however, after prolonged insult with high-fat diet animals enter a second, catabolic phase characterized by reduced bone mass, probably related with diet-induced alterations in metabolic profile of these obese mice (LeckaCzernik et al. 2015).

\section{The effect of the components of the HDL metabolic pathways on cartilage and bone}

\section{HDL and cartilage homeostasis}

A considerable volume of epidemiological studies has uncovered a strong connection between osteoarthritis (OA) and pathological conditions such as hypertension, dyslipidemia, coronary heart disease and type 2 diabetes. Therefore, it not surprising that several authors suggest that OA should be considered a facet of metabolic syndrome (Velasquez \& Katz 2010).

In symphony with the clinical data, molecular studies have shown that perturbations in lipid metabolic pathways are strongly related to the pathobiology of OA. Indeed, a sophisticated comparative proteome analysis on hypertrophied OA chondrocytes revealed that pathways regulating lipid metabolism, prostaglandin synthesis, glutathione metabolism and metabolism through the cytochrome p450, display differences between normal and hypertrophic OA chondrocytes (Tsolis et al. 2015). Moreover, gene expression analyses on human-derived OA chondrocytes uncovered disturbed expression levels of genes that regulate cholesterol influx and efflux and lipid metabolism (Tsolis et al. 2015). It has also been documented that the expression of a set of genes that regulate cholesterol efflux, namely ABCA1, APOA1 and LXR $\alpha$ is significantly suppressed in chondrocytes derived from OA in comparison to normal cartilage (Tsezou et al. 2010). Notably, after treatment with the LXR agonist TO-901317, the OA chondrocytes displayed augmented ABCA1 and APOA1 expression and cholesterol efflux (Tsezou et al. 2010). This finding is in line with a previous study by Collins-Racie and coworkers having examined 48 nuclear receptors and found that the expression levels of 23 of them had significant differences between normal and OA human cartilage samples (Collins-Racie et al. 2009). Interestingly, the mRNA of the nuclear receptors LXR $\alpha$ and LXR $\beta$ and their heterodimeric partners retinoid
$\mathrm{X}$ receptor (RXR) $\alpha$ and RXR $\beta$, as well as the LXR target genes ABCG1 and apolipoproteins D and E were greatly reduced in the $\mathrm{OA}$ compared to those in the normal cartilage, a finding proposing that the HDL metabolic pathway is deregulated in OA. Their finding that the LXR signaling alterations are restored with the use of the LXR agonist TO-901317 suggests LXR signaling modulation could add to the therapeutic armamentarium against OA (Collins-Racie et al. 2009).

Driven by the aforementioned data, and based on previous results of our research team on the role of the lipoprotein transfer system and HDL metabolic pathways in the pathogenesis of diet-induced obesity and nonalcoholic fatty liver disease (Karagiannides et al. 2008, Karavia et al. 2012), we were tempted to further explore the role of HDL in the pathobiology of OA. For this purpose, we applied histological, histomorphomentrical and molecular/biochemical methodologies and found that after the consumption of Western-type diet both LCAT- and APOA1-deficient mice developed OA, in contrast to the control groups (Triantaphyllidou et al. 2013). This suggests that alterations in HDL biogenesis and maturation predispose to the development of $\mathrm{OA}$ in mice after chronic insult with Western-type diet. Notably, we also observed that in sharp contrast to the LCAT $^{-/-}$mice, the APOA1 $1^{-/-}$were not quite as obese, an observation that raises the challenging possibility that altered HDL metabolism may have a direct destructive effect on articular cartilage that is independent of body weight and significantly contributes to the development of OA (Triantaphyllidou et al. 2013).

\section{HDL and bone homeostasis}

Recent data propose that there is an association between serum HDL levels and bone mass (Papachristou \& Blair 2016). However, the results that have been generated from epidemiological studies on humans are contradicting. Indeed, even though a considerable volume of these studies have shown that increased HDL is associated with better bone quality and reduced risk of osteoporosis, others support a negative relation between serum HDL levels and bone mass (Jeong et al. 2010, Ackert-Bicknell 2012, Li et al. 2015). It is believed that factors such as genetic background, age, dietary habits and metabolic status are responsible for this inconsistency. Therefore, research groups that work on this field have recruited experimental animal models in an effort to illuminate the molecular mechanisms that link HDL and bone homeostasis, 
as will be described in the following sections of the present review article.

The role of Scarb1 Several recent studies have investigated the possible connection between HDL and bone metabolism. Toward this direction the group of Moreau has extensively studied the role of the principal HDL receptor, the scavenger receptor class B, type I (SRBI), in the regulation of bone mass. SRBI is the product of the Scarb1 gene and binds HDL with high affinity, facilitating the transportation of cholesterol from peripheral organs to the liver (Acton et al. 1996), a process called reverse cholesterol transport.

In an elegant study, this group examined the phenotypic characteristics of femora obtained from Scarb1-deficient mice, in comparison to their wild-type counterparts (Martineau et al. 2014a,b). Serologic analysis revealed that the Scarb1 knockout mice had significantly elevated both total plasma and HDL cholesterol. Dynamic and static ( $\mu$ CT-based) histomorphometry uncovered that Scarb1 deficiency was followed by increased bone volume and elevated osteoblast number and function, particularly in the trabecular bone of 2-month-old female mice femora. Their in vitro approaches on MSC obtained from knockout (KO) and wild-type mice were in symphony with their histological and histomorphometrical results, further supporting the osteoblastogenic behavior of the Scarb1-null mice. SRB1 regulates the uptake of cholesterol at the adrenal glands, and thus, Scarb1-deficient mice are characterized by reduced glucocorticoid but elevated adrenocorticotropic hormone (ACTH) levels (Martineau et al. 2014a,b). Given that high concentrations of ACTH are anabolic for osteoblasts (Isales et al. 2010), this mechanism may possibly explain the high bone mass phenotype observed in the Scarb1 KO mice. Nevertheless, other mechanisms such as intrinsic cellular alterations of the canonical and non-canonical Wnt pathways that are not directly related to plasma HDL levels may explain the increased bone mass of Scarb1 KO mice (Martineau et al. $2014 a, b)$, further adding to the complexity of the relationship between HDL and bone metabolism.

The 'enigmatic' role of APOE ApoE is a $34.2 \mathrm{kDa}$ glycoprotein produced by the liver and other peripheral organs. Its main functions are atheroprotection and maintenance of plasma lipid homeostasis as it mediates the cellular uptake of chylomicron remnants, very lowdensity lipoprotein and low-density lipoprotein and their clearance from the circulation. Notably, APOE is also implicated in the de novo biosynthesis of HDL (Kypreos \& Zannis 2007). In a relatively recent study, investigating the genetic connection between HDL and bone mineral density (BMD), Ackert-Bicknell reported that APOE, along with PPAR $\gamma$, ESR1 and IL6, belongs to a set of genes that regulate both HDL and BMD (AckertBicknell 2012). Nevertheless, the role of APOE in bone pathophysiology still remains quite puzzling. Indeed, back in 2005, Schilling and coworkers, first published the results of a very interesting research study on the effects of APOE deficiency on bone mass. With the use of histological, histomorphometrical, biomechanical and in vitro approaches, the authors showed that the ApoEnull mice exhibit augmented osteoblastic function and increased rate of bone synthesis (Schilling et al. 2005). Previous in vitro and experimental animal-based studies have documented that APOE deficiency is associated with reduced uptake of vitamin K-containing lipoproteins by osteoblasts and that exogenous administration of APOE increases the uptake of chylomicron remnants that contain vitamin K (Newman et al. 2002, Niemeier et al. 2005). Taking into account that vitamin $\mathrm{K}$ is essential for osteocalcin carboxylation, the authors correctly surmised that impaired APOE results in enhanced bone mass, paralleling the phenotype of the osteocalcin-depleted mice (Niemeier et al. 2005). Nonetheless, this mechanism does not explain the whole phenotype of APOE deficiency as studies in mouse models have shown that shortage of osteocalcin is associated with augmented fat mass (Oldknow et al. 2015), in contrast to APOE deficiency that is characterized by reduced body fat content and smaller adipocytes (Huang et al. 2006). To further add to the complexity of the role of APOE in bone biology, a more recent study by the same group showed that the bone mass of APOE-null mice is substantially reduced after consumption of diabetogenic high-fat diet for 16 weeks. These knockout mice also have an interesting metabolic profile, characterized by normal weight and lowered levels of serum insulin, glucose and leptin (Bartelt et al. 2010). The aforementioned findings reinforce the prevailing notion that body weight per se cannot define the quality of bone; rather, it appears that the fine balance between local and systemic metabolic pathways is responsible for the determination of bone mass.

As mentioned previously, osteoblasts and lipoblasts originate from the same precursor, the bone marrow mesenchymal stem cell. Interestingly, APOE-deficient mice challenged with high fat display both reduced bone mass and bone marrow adiposity (Bartelt et al. 2010). This observation allows drawing the conclusion that lack

Published by Bioscientifica Ltd 
of APOE possibly halts bone marrow MSC maturation in early stages and thus affects both lipoblastic and osteoblastic lineage, through unidentified mechanisms. More recently, Dieckerman and coworkers performed biochemical, histomorphometrical and genetic analyses on transgenic mice and demonstrated that human APOE2 has the most significant effect on bone turnover, in comparison to the other natural human isoforms, namely APOE3 and APOE4. They also presented data introducing the APOE $\varepsilon 2$ allele as a latent risk factor for reduced trabecular bone mass and vertebrae fractures in humans (Dieckmann et al. 2013).

The role of APOE in the function of osteoclasts is also poorly understood. The studies of Bartelt and coworkers and Schilling and coworkers demonstrated that shortage of APOE does not influence the number or the function of osteoclasts in mice fed both high-fat or chow diet (Schilling et al. 2005, Bartelt et al. 2010). In sharp contrast, a more recent in vitro report on bone marrow-derived macrophages showed that APOE has an inhibitory effect on osteoclasts, which is mediated by c-FOS-, NFATc1- and NF-kB-related signaling cascades (Kim et al. 2013).

In aggregate, the current literature indicates that APOE serves as a potent regulator of mesenchymal stem cells and therefore affects bone mass; nevertheless, its role on osteoclastic function warrants further investigation.

The role of APOA1 APOA1 is a key molecule in the regulation of HDL biogenesis. Its role in the regulation of bone mass is graphically presented in Fig. 3. We have recently documented that in addition to its role in atheroprotection, APOA1 is also implicated in the development of non-alcoholic fatty liver disease as well as in the pathogenesis of osteoarthritis in mice (Karavia et al. 2012, Trantaphyllidou et al. 2013). To further explore whether HDL possesses a causative role in the regulation of bone mass, we studied the involvement of LCAT and APOA1 in the function of bone cells. For this purpose, we used a 12-week-old Lcat and ApoA1 KO male mice. Static and dynamic histomorphometrical analyses showed that the bone quality as well as the rate of new bone formation and the number of tartrate-resistant acid phosphatase (TRAP) positive cells were similar between the Lcat KO and the wild-type animals. Importantly, however, the ApoA1-/mice had significantly reduced bone mass in comparison with their wild-type counterparts, implying that it is the impaired synthesis and not the incomplete maturation of HDL that affects bone mass. Bone quality studies including Raman spectroscopy and three-point bending test revealed that these mice had a remarkable reduction of cross-linked collagen, which insinuates a significant deterioration of the biomechanical properties of femora obtained from these mice. Dynamic histomorphometry showed a significant reduction in calcein-labeled and double-labeled surfaces, but no differences in the TRAPpositive cells between the two groups. Through a series of in vitro and molecular experiments on mesenchymal stem cells, osteoblasts and osteoclasts isolated and cultured from femora of the two mouse groups, we uncovered that the osteoblast-related factors and signaling axes, RUNX2, osterix, COLLa1 and RANKL, were significantly impaired in the ApoA1 $\mathrm{KO}$ compared to those in the wild-type mice. On the contrary, genes that regulate osteoclastic differentiation and function namely TRAP (Acp5),
A

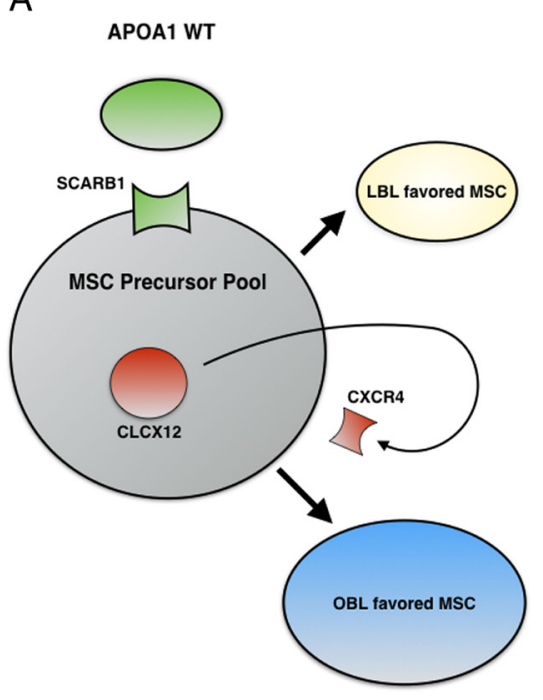

B

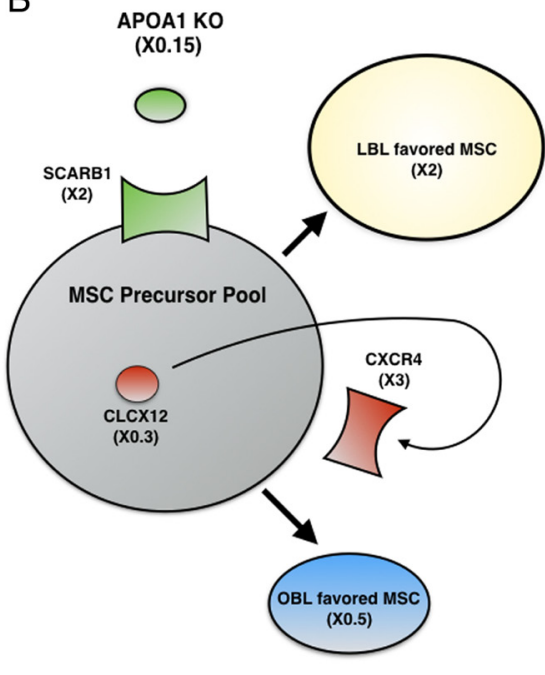

\section{Figure 3}

Diagram depicting the molecular changes observed in the ApoA-1-knockout mice (B) in comparison to their wild-type counterparts (A). All the molecular alterations are described in detail in the text. Note that the increased or decreased expression levels are represented by larger or smaller boxes. For example in the ApoA-1-deficient mice, the expression of CXCL12 is significantly decreased (XO.3), whereas the expression levels of its CXCL12 receptor CXCR4 is greatly elevated (X3). APOA1, Apolipoprotein A-1; CLCX12, CXC chemokine ligand 12; CXCR4, CXC Receptor 4; LBL, lipoblast; MSC, mesenchymal stem cells; OBL, osteoblast; WT, wild type. 
cathepsin K (Ctsk) and RANK (Tnfrsf11a) were unaffected (Blair et al. 2016a). These interesting findings indicate that the reduced bone mass observed in the ApoA1 KO mice is attributed primarily to suppressed osteoblastic bone synthesis and not to increased osteoclastic bone degradation.

It is well appreciated that bone marrow adiposity possesses an essential role in the regulation of osteoblasts and osteoclasts, and consequently, bone mass (LeckaCzernik \& Stechschulte 2014, Scheller et al. 2016). Histological examination of ApoA1 KO mice femora revealed that bone marrow of these animals have significantly elevated number of lipoblasts. In addition to the enhanced adiposity, the mesenchymal stem cells derived from the ApoA1-deficient mice exhibit increased expression levels of the lipoblast master regulators PPAR $\gamma$ and CEBPa (Blair et al. 2016a). Taking under consideration our finding that the number of bone marrow total mesenchymal stem cell population of both mouse groups was similar, we hypothesize that APOA1 may perform as a modulator of the mesenchymal stem cells pools within bone marrow, tipping the balance toward osteoblastogenesis (Blair et al. 2016a). The mechanisms that underline this phenomenon remain insufficiently elucidated.

One the most potent regulators of mesenchymal stem cell homing and bone synthesis is the CXC chemokine ligand 12 (CLCX12) that binds primarily to the receptor CXC receptor 4 (CXCR4) and is regulated, at least in part, by Annexin-2 (ANXA2) (Jung et al. 2011). We have very recently reported that APOA1 deficiency greatly disturbs the ANXA2-CLCX12-CXCR4 connection, providing a plausible mechanistic explanation for the impaired osteoblastic function observed in the ApoA1 KO mice (Blair et al. 2016a). It should also be borne in mind that the CLCX12-CXCR4 axis is also crucial for the regulation of osteoblastic bone marrow niche that participates in an array of processes, including hematopoietic stem cell (HSC) homing and mobilization and bone metastasis (Greenbaum et al. 2013, Kfoury \& Scadden 2015). Taken together, these reports fuel the attractive hypothesis that APOA1 may have a role in the pathobiology of HSC dormancy and/or motivation, and thus, it may be involved in the development of hematopoietic neoplasms. Moreover, given that reduced HDL levels are associated to increased metastasis rate (Pan et al. 2012), we advance the intriguing theory that perturbations in APOA1 may be implicated in the pathogenesis of bone metastases through alterations in signaling cascades and molecular axes, such as ANXA2-CLCX12-CXCR4 and
RANK-RANKL, which modify the microenvironment of the osteoblastic niche. Definitely, further studies are essential for the substantiation of these hypotheses.

\section{Conclusions and future perspectives}

As research on HDL progresses, new data in the literature strongly support a causative role of dysfunctional HDL with a number of metabolic disorders, including bone metabolic diseases (Constantinou et al. 2015, Papachristou $\&$ Blair 2016). Nonetheless, several critical issues should be addressed to unfold the 'mysteries' that underline the bone-HDL connection. First, the involvement of additional key regulators of the lipoprotein metabolism, such as ABCA1, LDLR and SRB1 in bone biology should be extensively assessed. The use of genetically modified mice is the best approach toward the salvation of these issues. Second, the role of bone marrow adiposity must be thoroughly examined. It is known that elevated marrow adiposity variably affects bone cells and is involved in the pathogenesis of bone-related pathologies such as osteoporosis and bone metastases. Moreover, novel data suggest that bone marrow microenvironment and the osteoblastic niche in particular are greatly influenced by HDL status. Taking into account that osteoblastic niche regulates the fine balance between dormancy and motivation of the HSCs and plausibly participates in the pathogenesis of hematopoietic neoplasias, it would be very intriguing to unveil the molecular cobblestone that link HDL metabolism to the development of these diseases. Finally, it should be kept in mind that HDL efficacy does not rely solely on the HDL-C levels, but also on the HDL particle functionality, which is defined by HDL apolipoprotein and lipid content (Filou et al. 2016). For this reason, the in-depth appreciation of the structure-function association of HDL will pave the way not only toward the thorough understanding of the mechanisms that link HDL and bone mass, but also toward the development of effective pharmaceuticals that will target HDL functionality. It is our expectation that the identification of surrogate markers of $\mathrm{HDL}$ functionality may prove invaluable for predicting the risk for developing bone metabolic disorders.

Obviously, many 'rivers need to be crossed' to achieve a proper understanding of the molecular basis of the complex HDL-bone interactions. However, it is now clear that the functional crosstalk between HDL and bone metabolism determines the beginning of a beautiful friendship that can potentially set the basis for

Published by Bioscientifica Ltd 
the development of novel HDL-directed pharmaceuticals for the treatment of bone pathologies.

\section{Declaration of interest}

The authors declare that there is no conflict of interest that could be perceived as prejudicing the impartiality of this review.

\section{Funding}

This work was supported in part by the Marie Curie Reintegration Grant (IRG), FP7-PEOPLE-RG-2009, (Grant number 256402-«apoeostearthritis»); the University of Patras, Faculty of Medicine KARATHEODORI Research Grants (Grant numbers D.155 and E.073); the 'ARISTIA I' of Hellenic GSRT (Grant number 248); the Department of Veteran's Affairs Grant BX002490 and by National Institutes of Health (USA) grants (grant numbers AR055208, and AR065407). This research study is an activity of the research network OsteoNet, http://www.osteonet.gr, of the University of Patras.

\section{References}

Ackert-Bicknell CL 2012 HDL cholesterol and bone mineral density: is there a genetic link? Bone $50525-533$. (doi:10.1016/j. bone.2011.07.002)

Acton S, Rigotti A, Landschulz KT, Xu S, Hobbs HH \& Krieger M 1996 Identification of scavenger receptor SR-BI as a high density lipoprotein receptor. Science 271 518-520. (doi:10.1126/ science.271.5248.518)

Barsh GS \& Schwartz MW 2002 Genetic approaches to studying energy balance: perception and integration. Nature Reviews Genetics $\mathbf{3}$ 589-600. (doi:10.1038/nrn902)

Bartelt A, Beil FT, Schinke T, Roeser K, Ruether W, Heeren J \& Niemeier A 2010 Apolipoprotein E-dependent inverse regulation of vertebral bone and adipose tissue mass in $\mathrm{C} 57 \mathrm{Bl} / 6$ mice: modulation by dietinduced obesity. Bone 47 736-745. (doi:10.1016/j.bone.2010.07.002)

Blair HC, Teitelbaum SL, Ghiselli R \& Gluck S 1989 Osteoclastic bone resorption by a polarized vacuolar proton pump. Science 245 855-857. (doi:10.1126/science.2528207)

Blair HC, Borysenko CW, Villa A, Schlesinger PH, Kalla SE, Yaroslavskiy BB, Garćia-Palacios V, Oakley JI \& Orchard PJ 2004 In vitro differentiation of CD14 cells from osteopetrotic subjects: contrasting phenotypes with TCIRG1, CLCN7, and attachment defects. Journal of Bone and Mineral Research 19 1329-1338. (doi:10.1359/ JBMR.040403)

Blair HC, Robinson LJ \& Zaidi M 2005 Osteoclast signalling pathways. Biochemical and Biophysical Research Communications 328 728-738. (doi:10.1016/j.bbrc.2004.11.077)

Blair HC, Schlesinger PH, Huang CL \& Zaidi M 2007 Calcium signalling and calcium transport in bone disease. Subcell Biochemistry $\mathbf{4 5}$ 539-562. (doi:10.1007/978-1-4020-6191-2_21)

Blair HC, Zaidi M, Huang CL \& Sun L 2008 The developmental basis of skeletal cell differentiation and the molecular basis of major skeletal defects. Biological Reviews Cambridge Philosophical Society 83 401-415. (doi:10.1111/j.1469-185X.2008.00048.x)

Blair HC, Kalyvioti E, Papachristou NI, Tourkova IL, Syggelos SA, Deligianni D, Orkoula MG, Kontoyannis CG, Karavia EA, Kypreos $\mathrm{KE}$, et al. 2016a Apolipoprotein A-1 regulates osteoblast and lipoblast precursor cells in mice. Laboratory Investigation 96 763-772. (doi:10.1038/labinvest.2016.51)

Blair HC, Larrouture QC, Li Y, Lin H, Beer Stoltz D, Liu L, Tuan RS, Robinson LJ, Schlesinger PH \& Nelson DJ $2016 b$ Osteoblast

http://joe.endocrinology-journals.org

DOI: 10.1530/JOE-16-0657
(C) 2017 Society for Endocrinology Printed in Great Britain differentiation and bone matrix formation in vivo and in vitro. Tissue Engineering Part B [in press]. (doi:10.1089/ten.TEB.2016.0454)

Bonewald LF 2011 The amazing osteocyte. Journal of Bone and Mineral Research 26 229-238. (doi:10.1002/jbmr.320)

Boyle WJ, Simonet WS \& Lacey DL 2003 Osteoclast differentiation and activation. Nature 423 337-342. (doi:10.1038/nature01658)

Brunham LR, Kruit JK, Iqbal J, Fievet C, Timmins JM, Pape TD, Coburn BA, Bissada N, Staels B, Groen AK, et al. 2006 Intestinal ABCA1 directly contributes to HDL biogenesis in vivo. Journal of Clinical Investigation 116 1052-1062. (doi:10.1172/JCI27352)

Burger D \& Dayer JM 2002 High-density lipoprotein-associated apolipoprotein A-I: the missing link between infection and chronic inflammation? Autoimmunity Reviews 1 111-117. (doi:10.1016/S15689972(01)00018-0)

Cao JJ 2011 Effects of obesity on bone metabolism. Journal of Orthopaedic Surgery and Research 6 30. (doi:10.1186/1749-799X-6-30)

Chroni A, Liu T, Gorshkova I, Kan HY, Uehara Y, von Eckardstein A \& Zannis VI 2003 The central helices of ApoA-I can promote ATPbinding cassette transporter A1 (ABCA1)-mediated lipid efflux. Amino acid residues 220-231 of the wild-type ApoA-I are required for lipid efflux in vitro and high density lipoprotein formation in vivo. Journal of Biological Chemistry 278 6719-6730. (doi:10.1074/jbc.M205232200)

Clevers H 2006 Wnt/b-catenin signaling in development and disease. Cell 127 469-480. (doi:10.1016/j.cell.2006.10.018)

Collins-Racie LA, Yang Z, Arai M, Li N, Majumdar MK, Nagpal S, Mounts WM, Dorner AJ, Morris E \& LaVallie ER 2009 Global analysis of nuclear receptor expression and dysregulation in human osteoarthritic articular cartilage: reduced LXR signaling contributes to catabolic metabolism typical of osteoarthritis. Osteoarthritis Cartilage 17 832-842. (doi:10.1016/j.joca.2008.12.011)

Constantinou C, Karavia EA, Xepapadaki E, Petropoulou PI, Papakosta E, Karavyraki M, Zvintzou E, Theodoropoulos V, Filou S, Hatziri A, et al. 2015 Advances in high-density lipoprotein physiology: surprises, overturns, and promises. American Journal of Physiology: Endocrinology and Metabolism 310 E1-E14. (doi:10.1152/ajpendo.00429.2015)

de Gorter DJJ \& ten Dijke P 2013 Signal transduction cascades controlling osteoblast differentiation. In Primer of the Metabolic Bone Diseases and Disorders of Mineral Metabolism, edn 8, pp 15-24. Ed CJ Rosen. Ames, IA, USA: John Wiley \& Sons, Inc. (doi:10.1002/9781118453926.ch2)

Delgado-Calle J, Sato AY \& Bellido T 2016 Role and mechanism of action of sclerostin in bone. Bone 96 29-37. (doi:10.1016/j. bone.2016.10.007)

Devlin RD, Reddy SV, Savino R, Ciliberto G \& Roodman GD 1998 IL-6 mediates the effects of IL-1 or TNF, but not PTHrP or 1,25(OH)2D3, on osteoclast-like cell formation in normal human bone marrow cultures. Journal of Bone and Mineral Research 13 393-399. (doi:10.1359/jbmr.1998.13.3.393)

Dieckmann M, Beil FT, Mueller B, Bartelt A, Marshall RP, Koehne T, Amling M, Ruether W, Cooper JA, Humphries SE, et al. 2013 Human apolipoprotein $\mathrm{E}$ isoforms differentially affect bone mass and turnover in vivo. Journal of Bone and Mineral Research 28 236-245. (doi:10.1002/jbmr.1757)

Eberhardt AW, Yeager-Jones A \& Blair HC 2001 Regional trabecular bone matrix degeneration and osteocyte death in femora of glucocorticoidtreated rabbits. Endocrinology 142 1333-1340. (doi:10.1210/ endo.142.3.8048)

Filou S, Lhomme M, Karavia EA, Kalogeropoulou C, Theodoropoulos V, Zvintzou E, Sakellaropoulos GC, Petropoulou PI, Constantinou C, Kontush A, et al. 2016 Distinct roles of apolipoproteins A1 and $\mathrm{E}$ in the modulation of high-density lipoprotein composition and function. Biochemistry 55 3752-3762. (doi:10.1021/acs. biochem.6b00389)

Fitzgerald ML, Morris AL, Chroni A, Mendez AJ, Zannis VI \& Freeman MW 2004 ABCA1 and amphipathic apolipoproteins form highaffinity molecular complexes required for cholesterol efflux. Journal of Lipid Research 45 287-294. (doi:10.1194/jlr.M300355-JLR200) 
Forlino A \& Marini JC 2016 Osteogenesis imperfecta. Lancet 3871657 1671. (doi:10.1016/S0140-6736(15)00728-X)

Gofman JW, Glazier F, Tamplin A, Strisower B \& De Lalla O 1954 Lipoproteins, coronary heart disease, and atherosclerosis. Physiological Reviews 34 589-607.

Greenbaum A, Hsu YM, Day RB, Schuettpelz LG, Christopher MJ, Borgerdling JN, Nagasawa T \& Link DC 2013 CXCL12 in early mesenchymal progenitors is required for haematopoietic stem-cell maintenance. Nature 495 227-230. (doi:10.1038/nature11926)

Havel RJ, Eder HA \& Bragdon JH 1955 The distribution and chemical composition of ultracentrifugally separated lipoproteins in human serum. Journal of Clinical Investigation 34 1345-1353. (doi:10.1172/ JCI103182)

Heller M, Stalder D, Schlappritzi E, Hayn G, Matter U \& Haeberli A 2005 Mass spectrometry-based analytical tools for the molecular protein characterization of human plasma lipoproteins. Proteomics 5 2619-2630. (doi:10.1002/pmic.200401233)

Huang X, Jiang Y \& Xia W 2013 FGF23 and phosphate wasting disorders. Bone Research 1 120-132. (doi:10.4248/BR201302002)

Huang ZH, Reardon CA \& Mazzon T 2006 Endogenous ApoE expression modulates adipocyte triglyceride content and turnover. Diabetes $\mathbf{5 5}$ 3394-3402 (doi:10.2337/db06-0354)

Isales CM, Zaid M \& Blair HC 2010 ACTH is a novel regulator of bone mass. Annals of the New York Academy of Sciences 1192 110-116. (doi:10.1111/j.1749-6632.2009.05231.x)

Jeong IK, Cho SW, Kim SW, Choi HJ, Park KS, Kim SY, Lee HK, Cho SH, Oh BH \& Shin CS 2010 Lipid profiles and bone mineral density in pre- and postmenopausal women in Korea. Calcified Tissue International 87 507-512. (doi:10.1007/s00223-010-9427-3)

Jung Y, Shiozawa Y, Wang J, Patel LR, Havens AM, Song J, Krebsbach PH, Roodman GD \& Taichman RS 2011 Annexin-2 is a regulator of stromal cell-derived factor-1/CXCL12 function in the hematopoietic stem cell endosteal niche. Experimental Hematology 39 151-166. (doi:10.1016/j.exphem.2010.11.007)

Kaneki H, Guo R, Chen D, Yao Z, Schwarz EM, Zhang YE, Boyce BF \& Xing L 2006 Tumor necrosis factor promotes Runx2 degradation through up-regulation of Smurf1 and Smurf2 in osteoblasts. Journal of Biological Chemistry 281 4326-4333. (doi:10.1074/jbc.M509430200)

Karagiannides I, Abdou R, Tzortzopoulou A, Voshol PJ \& Kypreos KE 2008 Apolipoprotein E predisposes to obesity and related metabolic dysfunctions in mice. FEBS Journal 275 4796-4809. (doi:10.1111/ j.1742-4658.2008.06619.x)

Karavia EA, Papachristou DJ, Liopeta K, Triantafyllidou IE, Dimitrakopoulos O \& Kypreos KE 2012 Apolipoprotein A-I modulates processes associated with diet-induced nonalcoholic fatty liver disease in mice. Molecular Medicine 18 901-912. (doi:10.2119/molmed.2012.00113)

Karavia EA, Zvintzou E, Petropoulou PI, Xepapadaki E, Constantinou C \& Kypreos KE 2014 HDL quality and functionality: what can proteins and genes predict? Expert Review of Cardiovascular Therapy 12 521-532. (doi:10.1586/14779072.2014.896741)

Karlsson H, Leanderson P, Tagesson C \& Lindahl M 2005a Lipoproteomics I: mapping of proteins in low-density lipoprotein using twodimensional gel electrophoresis and mass spectrometry. Proteomics 5 551-565. (doi:10.1002/pmic.200300938)

Karlsson H, Leanderson P, Tagesson C \& Lindahl M 2005b Lipoproteomics II: mapping of proteins in high-density lipoprotein using twodimensional gel electrophoresis and mass spectrometry. Proteomics 5 1431-1445. (doi:10.1002/pmic.200401010)

Kfoury Y \& Scadden DT 2015 Mesenchymal cell contributions to stem cell niche. Cell Stem Cell 16 239-253. (doi:10.1016/j.stem.2015.02.019)

Kim WS, Kim HJ, Lee ZH, Lee Y \& Kim HH 2013 Apolipoprotein E inhibits osteoclast differentiation via regulation of c-Fos, NFATc1 and NF-кB. Experimental Cell Research 319 436-446. (doi:10.1016/j. yexcr.2012.12.004)

Kobayashi K, Takahashi N, Jimi E, Udagawa N, Takami M, Kotake S, Nakagawa N, Kinosaki M, Yamaguchi K, Shima N, et al. 2000 Tumor

http://joe.endocrinology-journals.org

DOI: $10.1530 / \mathrm{JOE}-16-0657$
(C) 2017 Society for Endocrinology Printed in Great Britain necrosis factor $\alpha$ stimulates osteoclast differentiation by a mechanism independent of the ODF/RANKL-RANK interaction. Journal of Experimental Medicine 191 275-286. (doi:10.1084/jem.191.2.275)

Krieger M 2001 Scavenger receptor class B type I is a multiligand HDL receptor that influences diverse physiologic systems. Journal of Clinical Investigation 108 793-797. (doi:10.1172/JCI14011)

Krum SA, Chang J, Miranda-Carboni G \& Wang CY 2010 Novel functions for NFкB: inhibition of bone formation. Nature Reviews Rheumatology 6 607-611. (doi:10.1038/nrrheum.2010.133)

Kypreos KE 2008 ABCA1 promotes the de novo biogenesis of apolipoprotein CIII-containing HDL particles in vivo and modulates the severity of apolipoprotein CIII-induced hypertriglyceridemia. Biochemistry 47 10491-10502. (doi:10.1021/bi801249c)

Kypreos KE \& Zannis VI 2007 Pathway of biogenesis of apolipoprotein E-containing HDL in vivo with the participation of ABCA1 and LCAT. Biochemical Journal 403 359-367. (doi:10.1042/BJ20061048)

Kypreos KE, Gkizas S, Rallidis LS \& Karagiannides I 2013 HDL particle functionality as a primary pharmacological target for HDL-based therapies. Biochemical Pharmacology 85 1575-1578. (doi:10.1016/j. bcp.2013.03.004)

Lecka-Czernik B \& Stechschulte LA 2014 Bone and fat: a relationship of different shades. Archives of Biochemistry and Biophysics 561 124-129. (doi:10.1016/j.abb.2014.06.010)

Lecka-Czernik B, Stechschulte LA, Czernik PJ \& Dowling AR 2015 High bone mass in adult mice with diet-induced obesity results from a combination of initial increase in bone mass followed by attenuation in bone formation; implications for high bone mass and decreased bone quality in obesity. Molecular and Cellular Endocrinology 410 35-41. (doi:10.1016/j.mce.2015.01.001)

Li S, Guo H, Liu Y, Wu F, Zhang H, Zhang Z, Xie Z, Sheng Z \& Liao E 2015 Relationship of serum lipid profiles and bone mineral density in postmenopausal Chinese women. Clinical Endocrinology 82 53-58. (doi:10.1111/cen.12616)

Liu T, Krieger M, Kan HY \& Zannis VI 2002 The effects of mutations in helices 4 and 6 of ApoA-I on scavenger receptor class B type I (SR-BI)mediated cholesterol efflux suggest that formation of a productive complex between reconstituted high density lipoprotein and SR-BI is required for efficient lipid transport. Journal of Biological Chemistry 277 21576-21584. (doi:10.1074/jbc.M112103200)

Ma T, Miyanishi K, Suen A, Epstein NJ, Tomita T, Smith RL \& Goodman SB 2004 Human interleukin-1-induced murine osteoclastogenesis is dependent on RANKL, but independent of TNF- $\alpha$. Cytokine $\mathbf{2 6}$ 138-144. (doi:10.1016/j.cyto.2004.02.001)

Martin TJ \& Sims NA 2015 RANKL/OPG; critical role in bone physiology. Reviews in Endocrine and Metabolic Disorders 16 131-139. (doi:10.1007/ s11154-014-9308-6)

Martineau C, Kevorkova O, Brissette L \& Moreau R $2014 a$ Scavenger receptor class B, type I (Scarb1) deficiency promotes osteoblastogenesis but stunts terminal osteocyte differentiation. Physiological Reports 2 e12117. (doi:10.14814/phy2.12117)

Martineau C, Martin-Falstrault L, Brissette L \& Moreau R $2014 b$ The atherogenic Scarb1 null mouse model shows a high bone mass phenotype. American Journal of Physiology: Endocrinology and Metabolism 306 E48-E57. (doi:10.1152/ajpendo.00421.2013)

Miller GJ \& Miller NE 1975 Plasma-high-density-lipoprotein concentration and development of ischaemic heart-disease. Lancet $\mathbf{1}$ 16-19. (doi:10.1016/S0140-6736(75)92376-4)

Nagata KO, Nakada C, Kasai RS, Kusumi A \& Ueda K 2013 ABCA1 dimer-monomer interconversion during HDL generation revealed by single-molecule imaging. PNAS 110 5034-5039. (doi:10.1073/ pnas.1220703110)

Newman P, Bonello F, Wierzbicki AS, Lumb P, Savidge GF \& Shearer MJ 2002 The uptake of lipoprotein-borne phylloquinone (vitamin K1) by osteoblasts and osteoblast-like cells: role of heparan sulfate proteoglycans and apolipoprotein E. Journal of Bone and Mineral Research 17 426-433. (doi:10.1359/jbmr.2002.17.3.426) 
$\mathrm{Ng} \mathrm{A} \&$ Duque G 2010 Osteoporosis as a lipotoxic disease. BoneKEy 7 108-123. (doi:10.1138/20100435)

Niemeier A, Kassem M, Toedter K, Wendt D, Ruether W, Beisiegel U \& Heeren J 2005 Expression of LRP1 by human osteoblasts: a mechanism for the delivery of lipoproteins and vitamin K1 to bone. Journal of Bone and Mineral Research 20 283-293. (doi:10.1359/ JBMR.041102)

Okin PM, Hille DA, Wiik BP, Kjeldsen SE, Lindholm LH, Dahlöf B \& Devereux RB 2013 In-treatment HDL cholesterol levels and development of new diabetes mellitus in hypertensive patients: the LIFE Study. Diabetic Medicine 30 1189-1197. (doi:10.1111/dme.12213)

Oldknow KJ, MacRae VE \& Farquharson C 2015 Endocrine role of bone: recent and emerging perspectives beyond osteocalcin. Journal of Endocrinology 225 R1-R19. (doi:10.1530/JOE-14-0584)

Palagano E, Blair HC, Pangrazio A, Tourkova I, Strina D, Angius A, Cuccuru G, Oppo M, Uva P, Van Hul W, et al. 2015 Buried in the middle but guilty: intronic mutations in the tcirg1 gene cause human autosomal recessive osteopetrosis. Journal of Bone and Mineral Research 30 1814-1821. (doi:10.1002/jbmr.2517)

Pan B, Ren H, He Y, Lv X, Ma Y, Li J, Huang L, Yu B, Kong J, Niu C, et al. 2012 HDL of patients with type 2 diabetes mellitus elevates the capability of promoting breast cancer metastasis. Clinical Cancer Research 18 1246-1256. (doi:10.1158/1078-0432.CCR-11-0817)

Pandur P \& Kuhl M 2001 An arrow of wingless to take-off. Bioassays 23 207-210. (doi:10.1002/1521-1878(200103)23:3<207::AIDBIES1029>3.0.CO;2-0)

Papachristou DJ \& Blair HC 2016 Bone and high-density lipoprotein: beginning of a beautiful friendship. World Journal of Orthopedics $\mathbf{7}$ 74-77. (doi:10.5312/wjo.v7.i2.74)

Papachristou DJ, Papachroni KK, Basrda EK \& Papavassiliou AG 2009 Signaling networks and transcription factors regulating mechanotransduction in bone. Bioessays 31 794-804. (doi:10.1002/ bies.200800223)

Pixley FJ \& Stanley ER 2004 CSF-1 regulation of the wandering macrophage: complexity in action. Trends in Cell Biology 14 628-638. (doi:10.1016/j.tcb.2004.09.016)

Redlich K \& Smolen JS 2012 Inflammatory bone loss: pathogenesis and therapeutic intervention. Nature Reviews Drug Discovery 11 234-250. (doi:10.1038/nrd3669)

Reid IR 2008 Relationships between fat and bone. Osteoporosis International 19 595-606. (doi:10.1038/nrd3669)

Reid IR, Ames RW, Evans MC, Sharpe SJ \& Gamble GD 1994 Determinants of the rate of bone loss in normal postmenopausal women. Journal of Clinical Endocrinology and Metabolism 79 950-954. (doi:10.1210/jc.79.4.950)

Rezaee F, Casetta B, Levels JH, Speijer D \& Meijers JC 2006 Proteomic analysis of high-density lipoprotein. Proteomics 6 721-730. (doi:10.1002/pmic.200500191)

Scheller EL, Cawthorn WP, Burr AA, Horowitz MC \& MacDougald OA 2016 Marrow adipose tissue: trimming the fat. Trends in Endocrinology and Metabolism 27 392-403. (doi:10.1016/j.tem.2016.03.016)

Schilling AF, Schinke T, Münch C, Gebauer M, Niemeier A, Priemel M, Streichert T, Rueger JM \& Amling M 2005 Increased bone formation in mice lacking apolipoprotein E. Journal of Bone and Mineral Research 20 274-282. (doi:10.1359/JBMR.041101)

Schlesinger PH, Blair HC, Teitelbaum SL \& Edwards JC 1997 Characterization of the osteoclast ruffled border chloride channel and its role in bone resorption. Journal of Biological Chemistry 272 18636-18643. (doi:10.1074/jbc.272.30.18636)
Shah AS, Tan L, Long JL \& Davidson WS 2013 Proteomic diversity of high density lipoproteins: our emerging understanding of its importance in lipid transport and beyond. Journal of Lipid Research $\mathbf{5 4} 2575-2585$. (doi:10.1194/jlr.R035725)

Sims NA \& Gooi JH 2008 Bone remodeling: multiple cellular interactions required for coupling of bone formation and resorption. Seminars in Cell and Developmental Biology 19 444-451. (doi:10.1016/j. semcdb.2008.07.016)

Soutar AK, Garner CW, Baker HN, Sparrow JT, Jackson RL, Gotto AM \& Smith LC 1975 Effect of the human plasma apolipoproteins and phosphatidylcholine acyl donor on the activity of lecithin: cholesterol acyltransferase. Biochemistry 14 3057-3064. (doi:10.1021/bi00685a003)

Thompson WR, Rubin CT \& Rubin J 2012 Mechanical regulation of signaling pathways in bone. Gene 503 179-193. (doi:10.1016/j. gene.2012.04.076)

Timmins JM, Lee JY, Boudyguina E, Kluckman KD, Brunham LR, Mulya A, Gebre AK, Coutinho JM, Colvin PL, Smith TL, et al. 2005 Targeted inactivation of hepatic Abca1 causes profound hypoalphalipoproteinemia and kidney hypercatabolism of apoA-I. Journal of Clinical Investigation 115 1333-1342. (doi:10.1172/ JCI200523915)

Triantaphyllidou IE, Kalyvioti E, Karavia E, Lilis I, Kypreos KE \& Papachristou DJ 2013 Perturbations in the HDL metabolic pathway predispose to the development of osteoarthritis in mice following long-term exposure to western-type diet. Osteoarthritis Cartilage $\mathbf{2 1}$ 322-330. (doi:10.1016/j.joca.2012.11.003)

Trigatti BL, Krieger M \& Rigotti A 2003 Influence of the HDL receptor SR-BI on lipoprotein metabolism and atherosclerosis. Arteriosclerosis, Thrombosis, and Vascular Biology 23 1732-1738. (doi:10.1161/01. ATV.0000091363.28501.84)

Tsezou A, Iliopoulos D, Malizos KN \& Simopoulou T 2010 Impaired expression of genes regulating cholesterol efflux in human osteoarthritic chondrocytes. Journal of Orthopaedic Research $\mathbf{2 8}$ 1033-1039. (doi:10.1002/jor.21084)

Tsolis KC, Bei ES, Papathanasiou I, Kostopoulou F, Gkretsi V, Kalantzaki K, Malizos K, Zervakis M, Tsezou A \& Economou A 2015 Comparative proteomic analysis of hypertrophic chondrocytes in osteoarthritis. Clinical Proteomics 12 12. (doi:10.1186/s12014-015-9085-6)

Tsompanidi EM, Brinkmeier MS, Fotiadou EH, Giakoumi SM \& Kypreos KE 2010 HDL biogenesis and functions: role of HDL quality and quantity in atherosclerosis. Atherosclerosis 208 3-9. (doi:10.1016/j. atherosclerosis.2009.05.034)

Vaisar T, Pennathur S, Green PS, Gharib SA, Hoofnagle AN, Cheung MC, Byun J, Vuletic S, Kassim S, Singh P, et al. 2007 Shotgun proteomics implicates protease inhibition and complement activation in the antiinflammatory properties of HDL. Journal of Clinical Investigation 117 746-756. (doi:10.1172/JCI26206)

Van Eck M, Pennings M, Hoekstra M, Out R \& van Berkel TJ 2005 Scavenger receptor BI and ATP-binding cassette transporter A1 in reverse cholesterol transport and atherosclerosis. Current Opinion in Lipidology 16 307-315. (doi:10.1097/01.mol.0000169351.28019.04)

Velasquez MT \& Katz JD 2010 Osteoarthritis: another component of metabolic syndrome? Metabolic Syndrome and Related Disorders 8 295-305. (doi:10.1089/met.2009.0110)

Zannis VI, Kypreos KE, Chroni A, Kardassis D \& Zanni EE 2004 Biophysical analysis of apolipoprotein E3 variants linked with development of type III hyperlipoproteinemia. In Molecular Mechanisms of Atherosclerosis pp 111-174. Ed J Loscalzo. New York, NY, USA: Taylor \& Francis.

Received in final form 16 February 2017

Accepted 17 March 2017

Accepted Preprint published online 17 March 2017 http://joe.endocrinology-journals.org

DOI: $10.1530 / J O E-16-0657$
() 2017 Society for Endocrinology Printed in Great Britain
Published by Bioscientifica Ltd. 